\title{
Electrical Properties of PVA:PVP:PEG based Blend Polymer Electrolytes
}

\author{
M. Vahini, M. Muthuvinayagam, K. Sundaramahalingam
}

\begin{abstract}
This is a novel approach to get polyblend films with improved properties such as ionic conductivity and dielectric strength. Different ratios of PEG (5\%, 10\%, 15\%, 20\%, 25\%, 30\%) are added with optimized PVA: PVP ratio. PVA: PVP: PEG based BPEs are prepared by using solution casting method. The polymer PEG act as a plasticizer and chemically stable in air.The prepared electrolytes are characterized by XRD, FTIR and ionic conductivity studies. The XRD analysis shows amorphous nature of the polyblend electrolytes. Dielectric analysis is carried out for the BPEs. The higher conductivity is obtained for $20 \%$ PEG added polyblend electrolytes and it is 9.0x10-9 S/Cm. Thus, PVA: PVP: PEG (40:40:20) system is confirmed as optimized one for further studies to enhance the ionic conductivity.
\end{abstract}

Keywords: PVA, PVP, PEG, XRD, Conductivity, Polyblend electrolyte.

\section{INTRODUCTION}

$\mathrm{T}$ he polymer electrolytes system presents an unique opportunity for the future of energy storage like batteries, super capacitors, solar cell, and fuel cell and so on [1-2]. The improvement of polymer system is needed to develop the energy storage devices. So researchers are worked to get a new polymer system which having a high ionic conductivity, good electrical and mechanical strength [3]. Various methods are used to enhance the ionic conductivity of the polymer electrolytes such as block copolymers, blending polymers, addition of plasticizer, and addition of inert fillers and so on. Literature survey has revealed that most of the work reported on polyblend films such as PEO:PVA [3], PANI :PEG [4], combinations are attended to enhance the properties of polyblend films[5-6]. Synthesis of new materials with a wide variety of properties is possible by using the technique of Polymer blending. A physical mixture of different polymers is known as polymer blending [3]. It is a low cost way to produce a new material with using of already existing polymers. PVA is one of the polymer in this work because of it has a high dielectric and tensile strength, good in mechanical, electrical properties [7]. PVP is another polymer in the combination and it is selected due to good

Revised Manuscript Received on December 09, 2019.

* Correspondence Author

M. Vahini, International Research Centre, Kalasalingam Academy of Research and Education, Anand nagar, Krishnankoil-626126 tamilnadu, India. Email: mvahini1991@gmail.com

M. Muthuvinayagam*, Department of Physics, Kalasalingam Academy of Research and Education, Anand nagar, Krishnankoil-626126, tamilnadu, India. Email: mmuthuvinayagam@gmail.com

K. Sundaramahalingam, International Research Centre, Kalasalingam Academy of Research and Education, Anand nagar, Krishnankoil-626126 tamilnadu, India. Email: sundaramahalingam07@gmail.com environmental stability, easy process ability and moderate electrical conductivity [8]. The various ratio of PEG is added to PVA: PVP matrix. The added PEG can act as a plasticizer with film forming polymer and chemically stable in air [9].

In this present work, we have investigated the dielectric properties and ionic conductivity of prepared polyblend films. The prepared electrolytes are characterized by XRD, SEM and AC-impedance analysis.

\section{EXPERIMENTAL PROCEDURE}

Polymers PVA and PVP are maintained at equal ratio. Along with different ratio [47.5:47.5:05, 45:45:10, 42.5:42.5:15, 40:40:20 and 37.5:37.5:25] of PEG is added. Distilled water is used as solvent throughout the experiment. At room temperature, the samples have been prepared by first dissolving PVA in water and PVP is added to PVA solution with stirring until get homogeneous solution. Finally PEG is added to PVA:PVP solution and stirred to get a homogeneous solution. The prepared PVA:PVP:PEG solution was poured into Petri dishes and placed at room temperature without disturbance to evaporate the solvent. After 3 days, the films have been peeled from petri dishes.

\section{Characterization of the Polyblend ELECTROLYTES}

$\mathrm{X}$-ray diffraction pattern of the films was recorded using $\mathrm{Cu}$ $\mathrm{K} \alpha(\lambda=1.5406 \AA)$, Bruker made X-ray diffractometer. FTIR studies of the films were recorded using Shimadzu-IR Affinity-1 spectrometer instrument in the wavenumber range of $400 \mathrm{~cm}^{-1}-4000 \mathrm{~cm}^{-1}$. The morphology of the blend polymer electrolytes was carried with Carl ZEISS EVO 18 scanning electron microscope. Ionic conductivity measurement is made in the range of $42 \mathrm{~Hz}-1 \mathrm{MHz}$ at room temperature by using HIOKI 3532-50 LCR HI-TESTER Impedance analyzer.

\section{RESULTS AND DisCUSSION}

\section{A. XRD-studies}

The XRD pattern of prepared polyblend electrolytes is shown Fig.1. The XRD pattern of PVA:PVP:PEG blend polymer electrolytes show amorphous nature. While polymer PEG is added to the PVA:PVP electrolyte, the crystalline nature of the film decreases.

The broad peaks around 180 - 240 is due to semicrystalline polymer PVA and around 290 is due to presence of polymer PVP $[8,10]$. The addition of PEG polymer at various weight percent induces the decrease in intensity 


\section{Electrical Properties of PVA:PVP:PEG based Blend Polymer Electrolytes}

of PVA:PVP blend polymer electrolyte. The crystallinity of polymer electrolyte decreases which are observed by addition of PEG. PVA:PVP :PEG (40:40:20) system has maximum suppressed peak and increased amorphous nature.

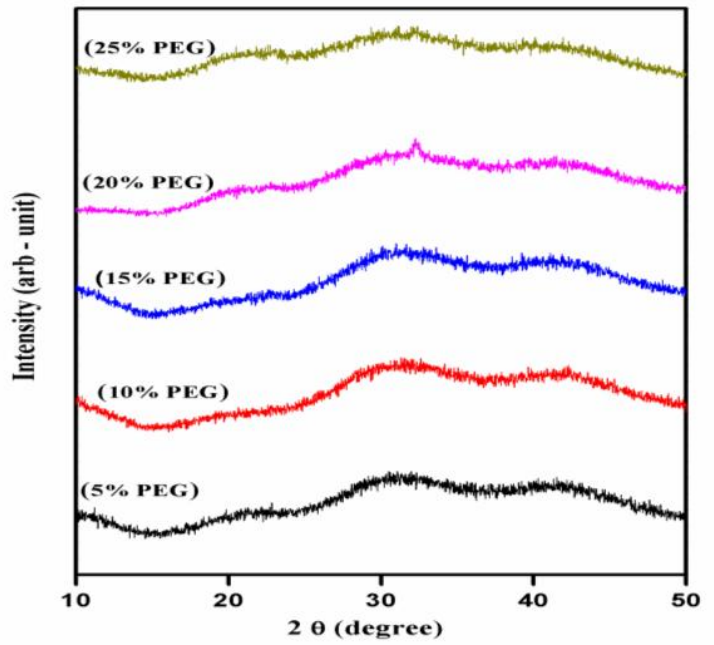

Fig.1 XRD pattern of PVA: PVP: PEG a)47.5:47.5:05
(b) $45: 45: 10$
(c) $42.5: 42.5: 15$
(d) 40:40:20 and (e)

$37.5: 37.5: 25$

\section{B. SEM-analysis}

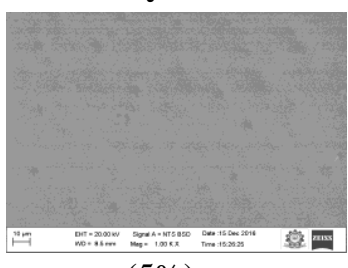

$(5 \%)$

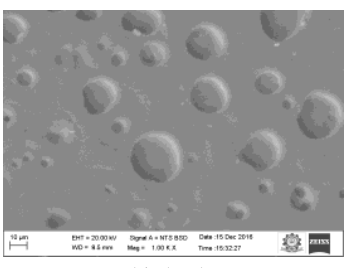

$(15 \%)$

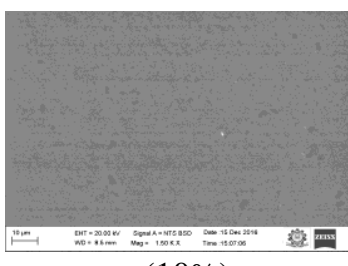

$(10 \%)$

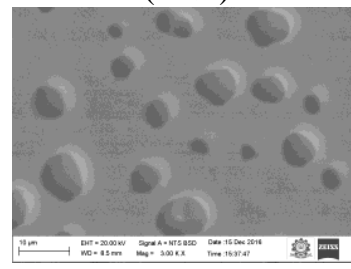

$(20 \%)$

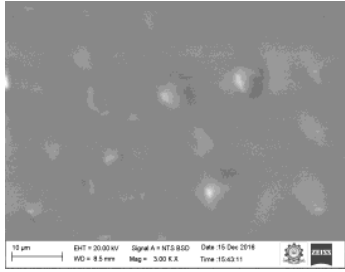

$(25 \%)$

Fig.2 SEM images of all polymer electrolytes with different ratios of PEG (5,10,15,20 and 25)

Fig. 2 shows SEM images of the polyblend electrolyte films. The miscibility of the polymers are clearly observed in the morphology images. The addition of PEG with PVA:PVP creates the porous nature which is used to enhance the disorderness [11]. The change in the morphology of the films are maximum observed for $20 \%$ of PEG added system. After that, the film has decreased disorderness which is coincident with XRD studies. Increase of smoothness reflects increase of amorphous nature. PEG has good compatibility and it can be mixed uniformly alongwith PVA:PVP polymer matrix [12].

\section{AC-Impedance studies}

\section{Conductivity studies}

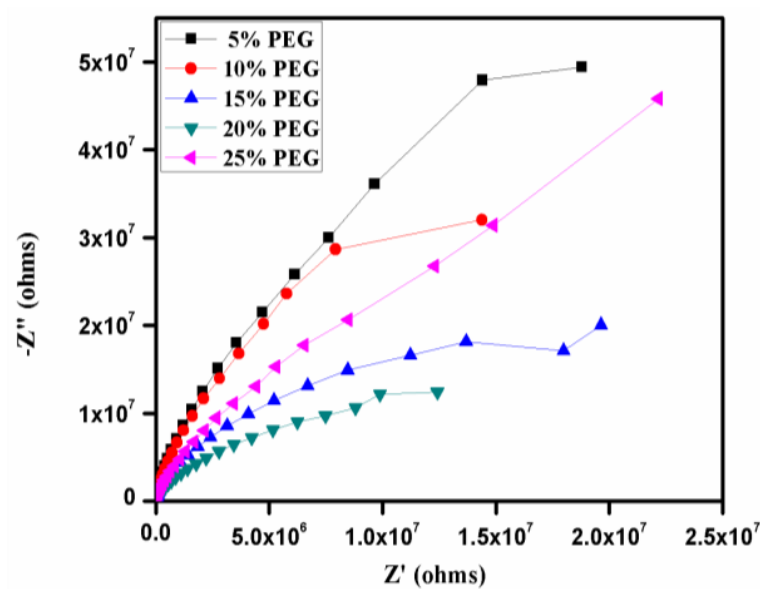

Fig.3 Cole-Cole plot of PVA:PVP:PEG polyblend films

The conductivity of the polyblend films are calculated by using bulk electrical resistance $(\mathrm{Rb})$, thickness $(\mathrm{t})$ and area (A) of the film. The relation among thickness, area and bulk electrical resistance is,

Conductivity ( , the value of $\mathrm{Rb}$ is extracted from Cole-Cole plot by using Z-view software. The Cole-Cole plot of the prepared polyblend films are shown in the Fig 3. The semicircle at high frequency is observed due to parallel combination of ionic migration and bulk polarization. Due to the solid state diffusion, the straight line is obtained at end of the semi-circle in Cole-Cole plot[13]. Among the system, the maximum conductivity is obtained for 40PVA:40PVP:20PEG polyblend film. The conductivity of the prepared system is shown in table.I

Table .I Conductivity values for prepared polymer electrolytes

\begin{tabular}{|c|c|}
\hline PVA: PVP: PEG system & Conductivity (S/cm) \\
\hline $47.5: 47.5: 05$ & $8.31 \times 10^{-10}$ \\
\hline $45: 45: 10$ & $1.06 \times 10^{-9}$ \\
\hline $42.5: 42.5: 15$ & $4.78 \times 10^{-9}$ \\
\hline $40: 40: 20$ & $9.00 \times 10^{-9}$ \\
\hline $37.5: 37.5: 25$ & $1.80 \times 10^{-9}$ \\
\hline
\end{tabular}

\section{Dielectric studies}

The amount of energy stored by materials which is explained by using dielectric studies. The dielectric response is generally denoted by the complex permittivity, $\varepsilon^{*}(\omega)$ $=\varepsilon^{\prime}(\omega)-\mathcal{E}^{\prime \prime}(\omega)$ where, $\varepsilon^{\prime}(\omega)$ - real and $\varepsilon^{\prime \prime}(\omega)$ - imaginary components storage and loss of energy. Both dielectric storage and loss are maximum at low frequency and relatively constant at high frequency.

The observed variations in dielectric spectrum which are due to the interfacial effects between electrode and electrolyte film $[13,15]$. Due to the inability of electric dipole to comply with of applied field, dielectric constant gets decreases as frequency increases [16]. Fig.4 and Fig.5 shows the dielectric constant and loss spectrum of prepared polyblend electrolytes. Due to the no excess ion diffusion at high frequency, the dielectric constant and loss 
gets decrease at high frequency region.

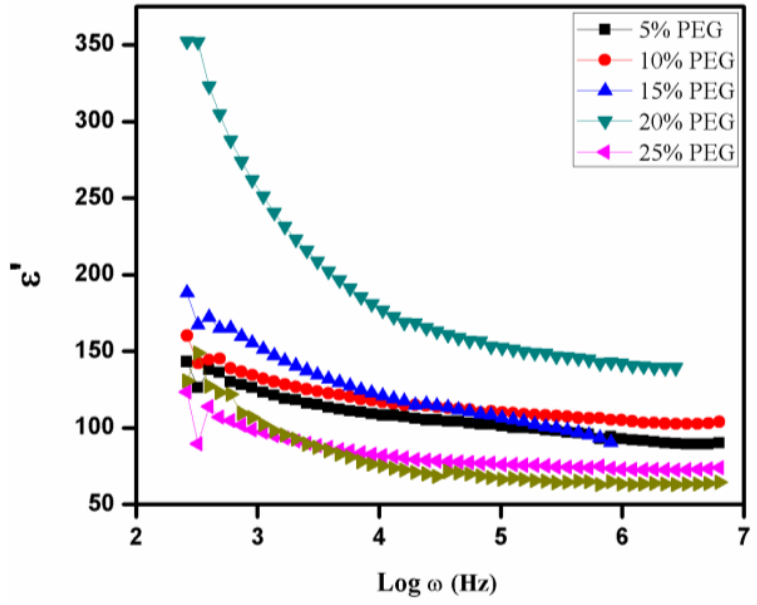

Fig.4 Dielectric constant spectra of PVA:PVP:PEG polyblend films

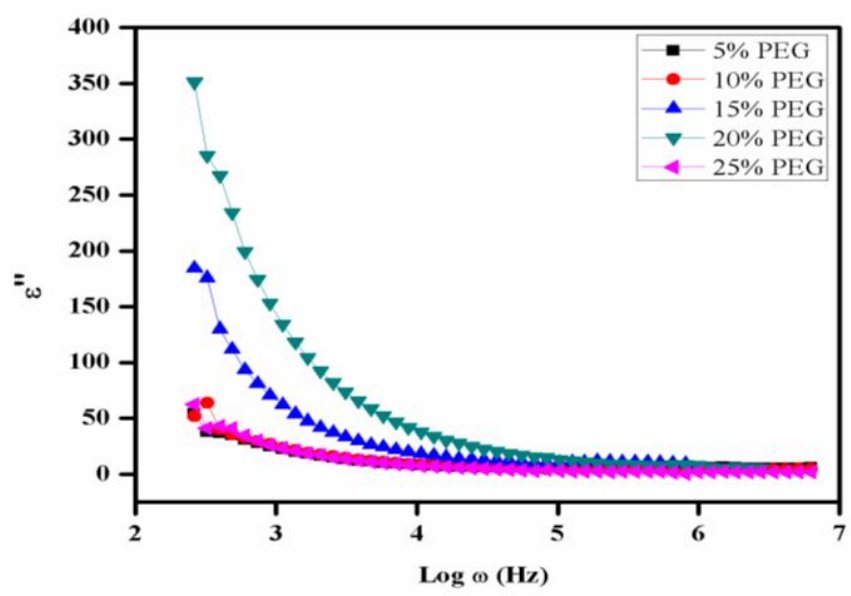

Fig.5 Dielectric loss spectra of PVA:PVP:PEG polyblend films

Modulus spectrum

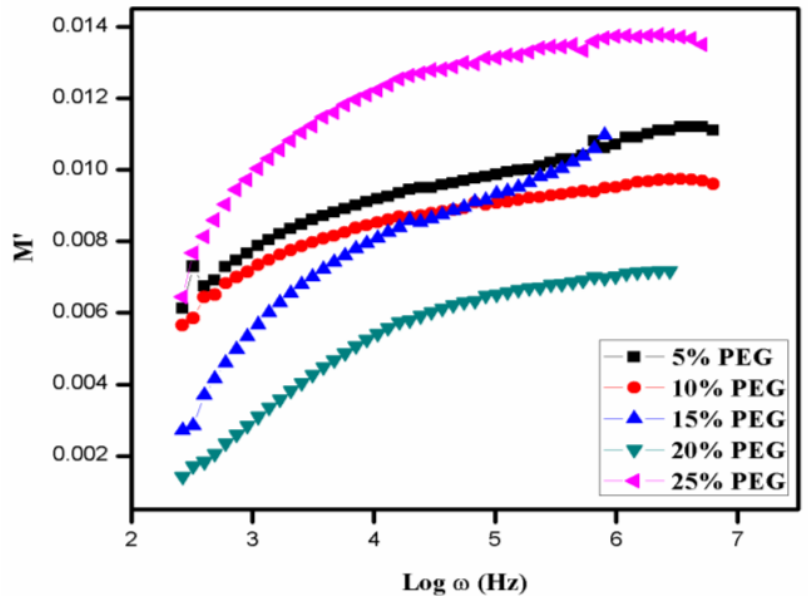

Fig.6 Real part of modulus spectra of PVA:PVP:PEG polyblend films

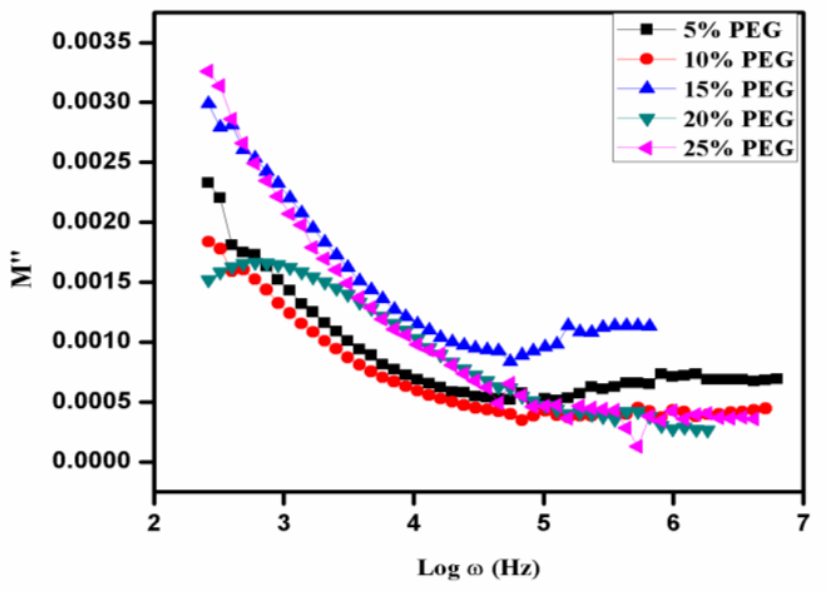

Fig.7 Imaginary part of modulus spectra of PVA:PVP:PEG polyblend films

Complex modulus spectra are shown in the Fig. 6 \& Fig.7. The interaction between electrode polarization and grain boundary can be discussed by using modulus spectrum. The complex modulus is generally denoted as $\mathrm{M}^{*}=\mathrm{M}^{\prime}+\mathrm{j} \mathrm{M}^{\prime \prime}$. The real $\left(\mathrm{M}^{\prime}\right)$ and imaginary $\left(\mathrm{M}^{\prime \prime}\right)$ parts of modulus can be expressed using dielectric permittivity. Due to the electrode polarization, the decreases in the $\mathrm{M}^{\prime}$ values at low frequency are obtained. The imaginary part of modulus has maximum value at low frequency and it gets decrease in the high frequency due to relaxation process in the system [17].

\section{Concentration dependent conductivity}

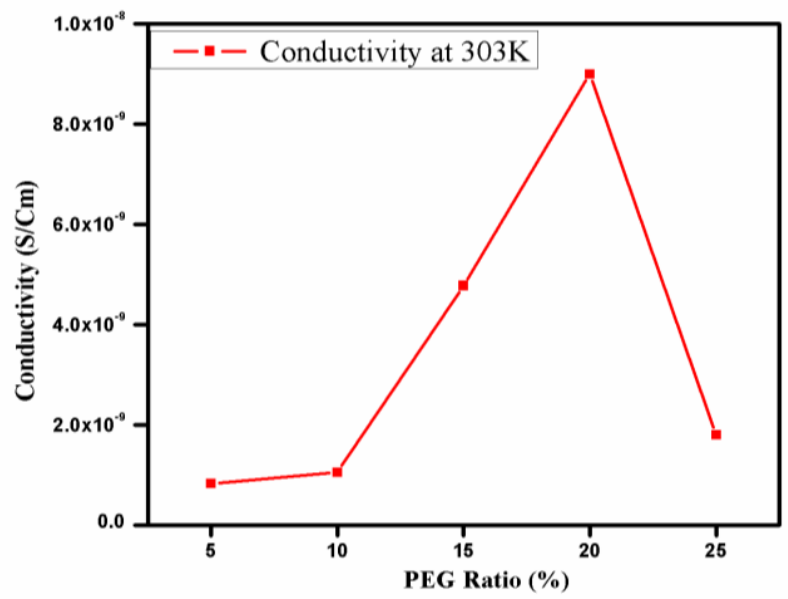

Fig.8 Conductivity with respect to concentration of PEG

The conductivity of the PVA:PVP polyblend film is enhanced by addition of PEG. The increased amorphous nature and enhanced smooth morphology is obtained by addition of $20 \%$ of PEG. It is confirmed in XRD studies and SEM analysis. Polymer PEG can act as a plasticizer. The presence of oxygen atom in polyether chain acts as an electron acceptor[15]. The dipolar molecules can be able to orient more easily in amorphous phase. The dielectric properties of the prepared systems are also improved with effect of PEG. The enhancement in the properties of the PVA:PVP polyblend film is achieved upto addition of 20\%PEG. The increase in conductivity of polyblend film with respect to concentration of PEG is shown in the Fig.8 


\section{Electrical Properties of PVA:PVP:PEG based Blend Polymer Electrolytes}

\section{CONCLUSION}

The solid polyblend electrolytes based on PVA, PVP with PEG was successfully prepared by solution casting technique and it is studied. Among the prepared systems, PVA: PVP: PEG (40:40:20) polyblend electrolyte is optimized by various characterization techniques such as XRD, SEM and AC-Impedance studies. The enhancement in the amorphous nature, conductivity, smooth morphology and dielectric properties of PVA:PVP polyblend films are achieved by addition of PEG polymer. The maximum conductivity of $9.00 \times 10-9 \mathrm{~S} / \mathrm{cm}$ is obtained for optimized polyblend electrolyte PVA:PVP:PEG (40:40:20) at room temperature. This optimized electrolyte can be futhur used to improve the ionic conductivity.

\section{ACKNOWLEDGMENT}

The authors acknowledge the management of Kalasalingam Academy of Research and Education, Krishnankoil-626126, for providing research facilities with research fellowship.

\section{REFERENCES}

1. K. S. Ngai, S. Ramesh, K. Ramesh, J. C. Juan. (2016) A review of polymer electrolytes: fundamental, approaches and applications, Ionics, 22 (8), 1259-1279.

2. B. Smitha, S. Sridhar, A.A. Khan. (2005) Solid polymer electrolyte membranes for fuel cell applications-a review, Journal of Membrane Science, 259, 10-26.

3. A. Chandra, Synthesis and dielectric studies of PEO-PVP blended solid polymer electrolytes, Indian Journal of Pure \& Applied Physics, 51 (2013), 788-791

4. P. Singh, Synthesis, (2014) Characterization and AC-Impedance Spectroscopic Studies on Interfacial Synthesis of Pani / PEG Nanocomposite, American Journal of Materials Science, 4(2), 74-83

5. B.K. Choi, Y.W. Kim, H.K. Shin. (2000) Ionic conduction in PEO-PAN blend polymer electrolytes, Electrochimica Acta, 45, 1371-1374.

6. T. Hamaya, S. Inoue, J. Qiao, T. Okada. (2006) Novel proton-conducting polymer electrolyte membranes based on PVA/PAMPS/PEG400 blend, Journal of Power Sources, 156, 311-314.

7. M. Hema, S. Selvasekarapandian, D. Arunkumar, A. Sakunthala, H. Nithya. (2009) FTIR, XRD and ac impedance spectroscopic study on PVA based polymer electrolyte doped with $\mathrm{NH}_{4} \mathrm{X}(\mathrm{X}=\mathrm{Cl}, \mathrm{Br}, \mathrm{I})$, Journal of Non-Crystalline Solids, 355, 84-90.

8. C. Venkata Subba Rao M. Ravi, V. Raja, P. BalajiBhargav, Ashok Kumar Sharma, V.V.R. Narasimha Rao. (2012) Preparation and characterization of PVP-based polymer electrolytes for solid-state battery applications, Iran Polym J., 21, 531-536.

9. G.K. Prajapati, P.N. Gupta. (2011) Comparative study of the electrical and dielectric properties of PVA-PEG- $\mathrm{Al}_{2} \mathrm{O}_{3}-\mathrm{MI}\left(\mathrm{M}^{1} / 4 \mathrm{Na}, \mathrm{K}, \mathrm{Ag}\right)$ complex polymer electrolytes, Physica $B, 406,3108-3113$.

10. K.M. Genova, S. Selvasekarapandian, S. Karthikeyan, N. Vijaya, S. Sivadevi, C. Sanjeeviraja. (2015) Lithium ion-conducting blend polymer electrolyte based on PVA-PAN doped with lithium nitrate, Polymer-Plastics Technology and Engineering, 55(1), 25-35.

11. Lizhen Fan, Zhimin Dang, Ce-Wen Nan, Ming Li. (2002) Thermal, electrical and mechanical properties of plasticized polymer electrolytes based on PEO/P(VDF-HFP) blends, Electrochimica Acta, 48, 205-209.

12. Jingyu Xi, Xinping Qiu, Jian Li, Xiao zhen Tang, Wentao Zhu, Liquan Chen. (2006) PVDF-PEO blends based microporous polymer electrolyte: Effect of PEO on pore configurations and ionic conductivity, Journal of Power Sources, 157, 501-506.

13. N. Rajeswari, S. Selvasekarapandian, S. Karthikeyan, M. Prabu, G. Hirankumar, H. Nithya, C. Sanjeeviraja. (2011) Conductivity and dielectric properties of polyvinyl alcohol-polyvinylpyrrolidone poly blend film using non-aqueous medium, Journal of Non-Crystalline Solids, 357, 3751-3756.
14. Arvind Awadhia, S.K. Patel, S.L. Agrawal. (2006) Dielectric investigations in PVA based gel electrolytes, Progress in Crystal Growth and Characterization of Materials, 52, 61-68.

15. Ch.V. Subba Reddy, Xia Han, Quan-Yao Zhu, Li-Qiang Mai, Wen Chen. (2006) Dielectric spectroscopy studies on (PVP + PVA) polyblend film, Microelectronic Engineering, 83, 281-285.

16. J.H. Joshi, D.K. Kanchan, M.J. Joshi, H.O. Jethva, K.D. Parikh. (2017) Dielectric Relaxation, Complex Impedance and Modulus Spectroscopic Studies of Mix Phase Rod like Cobalt Sulfide Nanoparticles, Material Research Bulletin, 93, 63-73.

\section{AUTHORS PROFILE}

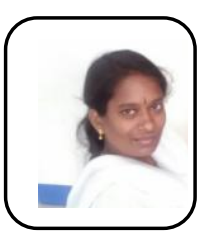

Dr. M. Vahini is a research scholar in the Department of Physics, School of Advanced sciences at Kalasalingam Academy of Research and Education, India. She obtained $\mathrm{PhD}$ in the area of polymer science from Kalasalingam Academy of Research and education at 2019 and M.Sc in Ayya Nadar Janaki Ammal college sivakasi and B.Sc at Sri Kaliswari college sivaksi. She has more experience in the polymer electrolyte for device application. She has published around seven peer-review research articles in journals.

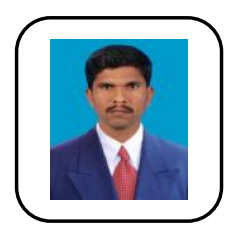

Dr. M. muthuvinayagam is an Associate professor in the Department of Physics, School of Advanced sciences at Kalasalingam Academy of Research and Education, India. He obtained $\mathrm{PhD}$ in the area of polymer science from Madurai Kamaraj University at 2016 and M.Sc and B.Sc from Ayya Nadar Janaki Ammal college sivakasi. He has more experience in the polymer electrolyte for device application. He has published more than ten peer-review research articles in journals.

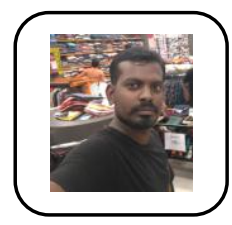

Mr. K. Sundaramahalingam is a Research scholar in the Department of Physics, School of Advanced Sciences at Kalasalingam Academy of Research and Education, India. He is doing his $\mathrm{PhD}$ in the area of polymer electrolyte for battery applications. He has obtained M.Sc Degree in Physics at Ayya Nadar Janaki Ammal College, sivakasi and B.Sc. Degree in physics from Arulmigu Kalasalingam college of Arts and Science at Krishnankoil. He has published five peer-review research articles. 